\title{
FIXED POINTS OF SUBOPEN MULTIFUNCTIONS
}

\author{
J. F. MCCLENDON
}

\begin{abstract}
A fixed point theorem of the Lefschetz type is proved for subopen (e.g., open-graph) multifunctions. A coincidence version is also given.
\end{abstract}

The fixed point theorem of $[5,6]$ is generalized here to fixed point theorems of the Lefschetz type. The following will be proved (see $\$ 1$ for terminology).

3.2 TheOREM. Let $X$ be a compact finite-dimensional $A N R$ and $m: X \rightarrow X a$ subopen multifunction with infinitely connected values. Then the Lefschetz number $L(m)=\sum_{q=0}^{\infty}(-1)^{q} \operatorname{tr}\left(m_{q}\right)$ is defined and if $L(m) \neq 0$ then $m$ has a fixed point.

3.3 Corollary. Let $X$ be an acyclic compact finite-dimensional $A N R$ and $m$ : $X \rightarrow X$ a subopen multifunction with infinitely connected values. Then $m$ has a fixed point.

The corollary includes the results of $[5,6]$, mentioned above. In $\$ 4$ a coincidence form of these is given.

1. Terminology, Lefschetz number. A set-valued function $m: X \rightarrow Y$ is called a multifunction if $m(x) \neq \varnothing$ for all $x$ in $X$. A point $x$ in $X$ is a fixed point of $m$ : $X \rightarrow X$ if $x \in m(x)$. The graph of $m: X \rightarrow Y$ is $G(m)=\{(x, y) \mid y \in m(x)\} \subset$ $X \times Y . m$ is called subopen if the projection $p: G(m) \rightarrow X$ can be locally embedded as an open subset of some Serre fibration, i.e., for each $x$ in $X$ there is an open nbhd $U$ of $x$ in $X$, a Serre fibration $q: E \rightarrow U$, and an embedding $e: p^{-1}(U) \rightarrow E$ such that $e\left(p^{-1}(U)\right)$ is open in $E$ and $q e=p$. A multifunction is an open-graph multifunction if $G(m)$ is open in $X \times Y$. So every open-graph multifunction is subopen.

A space $X$ is called $N$-connected if every map $\partial I^{j+1} \rightarrow X$ can be extended to a map $I^{j+1} \rightarrow X, 0 \leqslant j \leqslant N . X$ is infinitely connected if it is $N$-connected for all $N$. ANR means ANR (metric).

Homology will always be singular homology with coefficients in a fixed principal ideal domain $P$ (e.g., integers or any field) and $P$ will usually be omitted from the notation. See [11, p. 9] for a discussion of trace for a homomorphism $a: A \rightarrow A$ of finitely generated $P$-modules: $\operatorname{trace}(a)=\operatorname{tr}(a)$ is an element of $P$. For the spaces to be considered here, $H_{q}(X)$ will be a finitely generated $P$-module and will be zero except for a finite number of $q$. The induced homomorphism $m_{q}: H_{q}(X) \rightarrow H_{q}(X)$

Received by the editors January 6, 1981 and, in revised form, May 22, 1981.

1980 Mathematics Subject Classification. Primary 54H25. 
will be defined in $\$ 2$ for certain multifunctions $m: X \rightarrow X$. Then the Lefschetz number $L(m)=\Sigma(-1)^{q} \operatorname{tr}\left(m_{q}\right)$ (summation $q=0$ to $\infty$ always) is a well-defined element of $P$.

In [3] Eilenberg and Montgomery defined induced homomorphisms for certain closed-graph multifunctions (see [10] for more general versions). The procedure to be used here is analogous - the Vietoris mapping theorem is replaced by the Serre spectral sequence. To arrange the right setting some terminology will be helpful.

Say that a multifunction $m: X \rightarrow Y$ is a - - fibration multifunction, or simply a --- multifunction, if the projection $p: G(m) \rightarrow X$ is a ---- fibration. Recall that a map $E \rightarrow B$ is a Serre [Hurewicz] fibration if it has the homotopy lifting property for $C W$ complexes [all spaces]. Call $E \rightarrow B$ a Lefschetz fibration if it has the homotopy lifting property for compact ANR's. Thus we have Serre multifunctions, Hurewicz multifunctions, Lefschetz multifunctions, . . . .

It should be kept in mind that most of the multifunctions discussed in the literature (e.g., upper-semicontinuous, lower-semicontinuous) are not, in general, fibration multifunctions at all. Even a subopen multifunction (often a special case of lower-semicontinous multifunctions) is not a fibration multifunction in general. Michael's results [8, Proposition 4.1; 9, Corollary 8.4] can be viewed as giving sufficient conditions for a continous multifunction to be, respectively, a Serre multifunction or a Hurewicz multifunction. Other selection theorems in the literature are open to a similar interpretation.

A space $X$ is acyclic if it is path connected and $H_{q}(X)=0$ for all $q \geqslant 1$.

Assume spaces are nonempty.

A map $E \rightarrow B$ is an $N$-fibration if it has the homotopy lifting property for $C W$ complexes of $\operatorname{dim} \leqslant N$. For the reader's convenience the following result is stated.

[7, LEMMA 1.2] Let $T \rightarrow B$ be a Serre fibration and $E$ open in $T$. Assume each $E(b)$ is $N$-connected and nonempty. Then $E \rightarrow B$ is an $N$-fibration.

\section{Induced homomorphisms.}

2.1 LemMA. Assume $m: X \rightarrow Y$ is a Serre multifunction with acyclic values. Then

(1) $m_{q}: H_{q}(X ; A) \rightarrow H_{q}(Y ; A)$ is a (single-valued) homomorphism for all $q$ and any $P$-module $A$.

(2) If $f: X \rightarrow Y$ is a continuous selection for $m$ then $m_{q}=f_{q}$.

Proof. $p: G(m) \rightarrow X$ is a Serre fibration with fiber $p^{-1}(x)=x \times m(x) \equiv m(x)$ which is acyclic by hypothesis. So by $\left[11\right.$, pp. 476, 506] $p_{q}: H_{q}(G(m) ; A) \rightarrow$ $H_{q}(X ; A)$ is isomorphic for all $q$ and any $P$-module $A$. (Spanier [11] assumes the base is path-connected but in our situation $p: G(m) \rightarrow X$ is onto with path-connected fiber so the result extends easily to an arbitrary base.) Let $r: G(m) \rightarrow Y$ be the projection and define $m_{q}=r_{q} p_{q}^{-1}: H_{q}(X ; A) \rightarrow H_{q}(Y ; A)$.

Suppose a map $f: X \rightarrow Y$ is a selection for $m$. Let $f^{\prime}(x)=(x, f(x))$. Then $f^{\prime}$ : $X \rightarrow G(m)$ is a section for $p$ and $p_{*}^{-1}=f_{*}^{\prime}$. Also $r f^{\prime}=f$ so $m_{*}=r_{*} p_{*}^{-1}=r_{*} f_{*}^{\prime}=$ $\left(r f^{\prime}\right)_{*}=f_{*}$. 
Note that if $X$ and $Y$ are path-connected them $m_{0}=$ the identity : $A \rightarrow A$. Henceforth we will take $A=P$ and omit it from the notation. Note that the statements of the fixed point results in 3.1 and 3.4 make sense if "infinitely connected values" is replaced by the weaker "acyclic values". I do not know whether or not the results are then valid.

\section{Fixed point theorems.}

3.1 TheOREM. Suppose $X$ is a compact finite-dimensional ANR and $m: X \rightarrow X a$ Serre multifunction with infinitely connected values. Then $L(m)=\Sigma(-1)^{q} \operatorname{tr}\left(m_{q}\right)$ is defined and if $L(m) \neq 0$ then $m$ has a fixed point.

Proof. Lemma 2.1 shows $L(m)$ defined. By [1, p. 122] a compact finite-dimensional ANR is a retract of a compact polyhedron so by an easy argument any Serre fibration will have the homotopy lifting property for compact finite-dimensional ANR's. By hypothesis $G(m) \rightarrow X$ is a surjective Serre fibration with infinitely connected fiber so standard obstruction theory (e.g., [11, Theorem 22, p. 404; Theorem 9, p. 416]) gives a cross section $s: X \rightarrow G(m), p s=1$. Then $f=r s$ is a selection for $m$, so by $2.1, L(f)=L(m) \neq 0$. The classical Lefschetz theorem (e.g., $[11$, p. 195; 2, p. 43], the proofs are valid over $P$ ) shows that $f$ has a fixed point so $m$ does also.

3.2 TheOREM. Let $X$ be a compact finite-dimensional $A N R$ and $m: X \rightarrow X a$ subopen multifunction with infinitely connected values. Them $L(m)=\Sigma(-1)^{q} \operatorname{tr}\left(m_{q}\right)$ is defined and if $L(m) \neq 0$ then $m$ has a fixed point.

Proof. [7, Lemma 1.2] shows that $G(m) \rightarrow X$ is locally a Serre fibration and it follows easily that it is a Serre fibration. Thus $m$ is a Serre multifunction and 3.2 follows from 3.1 .

3.3 Corollary. Let $X$ be an acyclic compact finite-dimensional $A N R$ and $m$ : $X \rightarrow X$ a subopen multifunction with infinitely connected values. Then $m$ has a fixed point.

Proof. Since $H_{q}(X)=0, q>0$, and $X$ is path-connected, $m_{q}=0, q>0$, and $m_{0}=$ id: $P \rightarrow P$. Thus $L(m)=1 \neq 0$ and 3.2 gives a fixed point.

Notes. (1) 3.3 applies to a compact finite-dimensional AR. (2) Suppose the $m$ : $X \rightarrow X$ in 3.3 is open-graph and has only $N$-connected values. If we assume $X$ a compact ANR of dimension $\leqslant N$ then the values are ANR's of dimension $<N$ (since open) and since they are $N$-connected they are contractible-so the above results apply.

3.4. TheOREM. Let $X$ be a compact ANR and $m: X \rightarrow X$ a Lefschetz multifunction with infinitely connected values. Then $L(m)=\Sigma(-1)^{q} \operatorname{tr}\left(m_{q}\right)$ is defined and if $L(m) \neq$ 0 then $m$ has a fixed point.

Proof. The proof is the same as that of Theorem 3.1. 
4. Coincidence theorem. If $f, g: Z \rightarrow X$ are maps then they have a coincidence if there is a $z$ in $Z$ with $f(z)=g(z)$. A map $g: Z \rightarrow X$ is called subopen if it can be locally embedded as an open subset of a Serre fibration.

4.1 TheOREM. Let $X$ be a compact acyclic polyhedron of dimension $<N$ [or a retract of such a polyhedron]. Suppose $f, g: Z \rightarrow X$ are maps and $g$ is subopen with each $g^{-1}(x) N$-connected. Then $f$ and $g$ have a coincidence.

Proof. It follows from [7, Lemma 1.2] that $g: Z \rightarrow X$ is an $N$-fibration. Let $F=g^{-1}(x)$ for some fixed $x . g_{*}: \pi_{q}(Z) \rightarrow \pi_{q}(X)$ is the composite of $j_{*}: \pi_{q}(Z) \rightarrow$ $\pi_{q}(Z, F)$ and $\bar{g}_{*}: \pi_{q}(Z, F) \rightarrow \pi_{q}(X, *)=\pi_{q}(X)$. Since $F$ is $N$-connected the exact sequence of the pair $(Z, F)$ shows that $j_{*}$ is isomorphic for $q<N$ and onto if $q=N+1$. The proof of [4, Theorem 11.8, p. 83] shows that $\bar{g}_{*}$ is also isomorphic for $q \leqslant N$ and onto for $q=N+1$ (since $Z \rightarrow X$ is an $N$-fibration).

By [11, Theorem 22, p. 404], since $g: Z \rightarrow X$ is an $(N+1)$-equivalence, there is an $s: X \rightarrow Z$ with $g s \sim$ id. Since $Z \rightarrow X$ is an $N$-fibration and $\operatorname{dim}(X)<N$ we get $s^{\prime}: X \rightarrow Z$ with $g s^{\prime}=$ id [i.e., if $H: g s \sim$ id then $H$ lifts to $\bar{H}: X \times I \rightarrow Z$ with $\bar{H}_{0}=s, s^{\prime}=\bar{H}_{1}$ works]. Now $h=f s^{\prime}: X \rightarrow X$ has a fixed point $x$ by the classical Lefschetz theorem, $x=h(x)=f s^{\prime}(x)$. Set $z=s^{\prime}(x)$. Then $f(z)=x$ and also $g(z)=$ $g s^{\prime}(x)=x$. Q.E.D.

Note. By using $Z=G(m)$ and $N=\infty$ we see that 4.1 includes 3.3. If $X$ is a compact polyhedron of dimension $\leqslant N$ then $L(f, g)$ can be defined as $L(f s)$ giving a coincidence form of 3.1 .

\section{REFERENCES}

1. K. Borsuk, Theory of retracts, Polish Scientific Publishers, Warsaw, 1967.

2. R. Brown, The Lefschetz fixed point theorem, Scott, Foresman \& Co., Glenview, Illinois, 1971.

3. S. Eilenberg and D. Montgomery, Fixed point theorems for multivalued transformations, Amer. J. Math. 58 (1946), 214-222.

4. B. Gray, Homotopy theory, Academic Press, New York, 1975.

5. A. Mas Colell, A selection theorem for open graph correspondences with star-shaped values, J. Math. Anal. Appl. 68 (1979), 273-275.

6. J. McClendon, Note on a selection theorem of Mas Colell, J. Math. Anal. Appl. 77 (1980), 326-327.

7. Subopen multifunctions and selections (submitted).

8. E. Michael, Continuous Selections. III, Ann. of Math. 65 (1957), 375-390.

9. __ Convex structures and contimuous selections, Canad. J. Math. 11 (1959), 556-575.

10. M. Powell, Multivalued mappings and Lefschetz fixed point theorems, Proc. Cambridge Philos. Soc. 68 (1970), 619-630.

11. E. Spanier, Algebraic topology, McGraw-Hill, New York, 1966.

Department of Mathematics, University of Kansas, Lawrence, Kansas 66045 